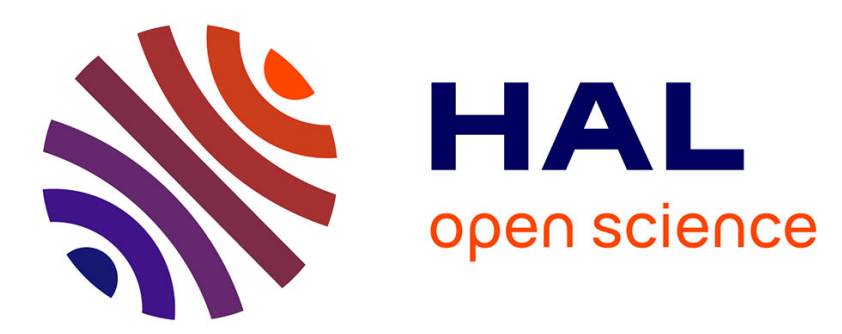

\title{
Mettre en scène Twitter dans les émissions politiques télévisées : pratique journalistique hybride et construction artefactuelle d'une " opinion publique connectée"
}

Michael Nexon

\section{To cite this version:}

Michael Nexon. Mettre en scène Twitter dans les émissions politiques télévisées: pratique journalistique hybride et construction artefactuelle d'une "opinion publique connectée ". Les Enjeux de l'information et de la communication, 2018. halshs-01977602

\section{HAL Id: halshs-01977602 \\ https://shs.hal.science/halshs-01977602}

Submitted on 10 Jan 2019

HAL is a multi-disciplinary open access archive for the deposit and dissemination of scientific research documents, whether they are published or not. The documents may come from teaching and research institutions in France or abroad, or from public or private research centers.
L'archive ouverte pluridisciplinaire HAL, est destinée au dépôt et à la diffusion de documents scientifiques de niveau recherche, publiés ou non, émanant des établissements d'enseignement et de recherche français ou étrangers, des laboratoires publics ou privés. 


\title{
Mettre en scène Twitter dans les émissions politiques télévisées : pratique journalistique hybride et construction artefactuelle d'une « opinion publique connectée »
}

\author{
Staging Twitter in political television programs: hybrid journalistic practice and artifactual \\ construction of a "connected public opinion"
}

Escenificar Twitter en los programas políticos de televisión: práctica periodística híbrida y construcción artefactual de una "opinión pública conectada"

Article inédit, mis en ligne le 31 octobre 2018.

\section{Michael NEXON}

Michaël Nexon est doctorant à l'université Lyon 2 sous la direction de Julia Bonaccorsi, co-encadré par Annelise Touboul et rattaché au laboratoire Elico. Ses travaux portent notamment sur les variations intermédiatiques que proposent aujourd'hui les émissions politiques télévisées qui intègrent des formes de dialogisme avec certains réseaux socionumériques comme Facebook ou plus régulièrement Twitter. Mail : michael-nexon@hotmail.fr

\section{Plan de l'article}

Introduction

$1 /$ Croquer une " opinion publique connectée » : objectivation et expertise journalistique Pluralité des objectifs et appréhension de Twitter comme espace protéiforme Le chiffre comme approbation d'un succès commercial et démocratique Le fact-checking ou comment s'instituer comme autorité énonciative Recourir au « micro-twittoir » : le journaliste comme porte-parole d'une vox populi

2/ Croquer le politique : de l'expertise à l'info-divertissement Le tweet comme petite phrase Mobiliser l'humour pour rejouer les codes de l'émission Rituel d'inversion et mise à l'épreuve de l'invité politique

Conclusion

Références bibliographiques

\section{Résumé}

Cet article vise à comprendre la manière dont les journalistes politiques - bousculés dans leur identité, leur centralité et leurs pratiques professionnelles par les réseaux socionumériques - se saisissent de discours produits sur Twitter comme éléments objectifs d'une opinion publique «connectée». Or, si cette dernière ne peut être scientifiquement prouvée, elle trouve dans les discours journalistiques une évidence qui empêche toute remise en question. Mettre en scène cette «opinion publique» permet surtout de replacer le journaliste dans une position magistrale et 
hybride, entre expertise et info-divertissement. Notre analyse sémio-pragmatique et discursive porte sur l'émission Des paroles et des actes et plus particulièrement la séquence finale dédiée aux réactions des internautes animée par un journaliste « expert » des réseaux socionumériques chargé de relayer la parole et l'opinion des « Français».

\section{Mots clés}

Journalisme politique, Twitter, opinion publique connectée, variation intermédiatique, discours.

\section{Abstract}

This article aims at understanding the way political journalists - whose identity, centrality and professional practices are totally changed by social networking sites - use the discourses produced on Twitter as objective insights from a "connected" public opinion. While this "public opinion" cannot be scientifically proven, it is presented as unquestionable in the journalistic discourse. By stageing this "public opinion", journalists hold a masterful and hybrid position that sits between that of expertise and info-divertissement. Our semio-pragmatic and discourse analysis will focus on the French political television program Des paroles et des actes and, in particular, on the final sequence dedicated to feedback from Internet users. The sequence is presented by a journalist "specialized" in social networking sites, responsible for passing on the words and opinion of "the French people".

\section{Keywords}

Political journalism, Twitter, connected public opinion, inter-media variation, discourse

\section{Resumen}

Este artículo apunta a entender cómo los periodistas -cuya identidad, centralidad y prácticas profesionales están cambiando por completo por los sitios de redes sociales- utilizan los tecnodiscursos producidos en Twitter como elementos objetivos de una opinión pública "conectada". Esta opinión pública no puede ser científicamente comprobada; sin embargo, el discurso periodístico no la cuestiona. Escenificar esta "opinión pública" permite ante todo colocar al periodista en una postura magistral e híbrida, entre pericia e infotainment. Nuestro análisis semiopragmático y discursivo tratará del programa político de televisión francés Des paroles et des actes $\mathrm{y}$, más particularmente, de la secuencia final en la que se escuchan las reacciones de los internautas. Esta secuencia es animada por un periodista "experto" en las redes sociales, encargado de transmitir la palabra y la opinión de "los Franceses".

Palabras clave

Periodismo político, Twitter, opinión pública conectada, variación inter-mediática, discurso 


\section{Introduction}

Si la médiation télévisuelle du politique n’est pas nouvelle (Leroux et Riutort, 2013 ; Neveu, 1995), force est de constater une évolution récente de ses cadres de représentation avec la mise en place de dispositifs intermédiatiques qui offrent aux téléspectateurs la possibilité de commenter les émissions sur les réseaux socionumériques Facebook et Twitter et de voir une sélection de discours apparaître dans le cadre télévisuel. Des Paroles et des actes ou encore L'Emission politique ont également intégré dans leur trame une séquence dédiée aux réactions des internautes, animée par un journaliste « expert » des réseaux socionumériques chargé de relayer la parole et l'opinion des « Français ».

Les réseaux socionumériques sont mobilisés tels des espaces publics à l'intérieur desquels se fabrique et circule de l'information politique jugée pertinente et dont il faudrait rendre compte notamment à travers une «revue de tweets». Si ces dispositifs permettent de produire du commentaire à partir des émissions politiques, celles-ci cherchent aussi à en tirer du bénéfice en produisant leur propre commentaire. Un jeu de médiation-remédiation des énoncés - des normes langagières et visuelles propres à l'espace numérique - se met en place et nourrit la pratique journalistique.

L'enjeu serait ici de rendre compte d'une «opinion publique ». Certains discours journalistiques tendent à proposer sous la forme de l'évidence un triptyque équationnel entre opinion publique, internautes et réseaux socionumériques alors même que ceux-ci doivent être pensés dans leur spécificité propre. Julien Boyadjian (2014) montre que les utilisateurs de Twitter qui produisent de la discursivité politique ne peuvent être envisagés comme représentatifs de l'opinion publique. Ils sont plus jeunes, plus masculins et plus diplômés que la population française.

Cet article ne réclame nullement de donner une définition de ce que serait une opinion publique " connectée». La question n'est pas de savoir si cette opinion publique peut être acceptable d'un point de vue scientifique. A la suite des travaux de Luc Boltanski (1991), nous pensons que ce n'est pas tant la réalité objective qui compte que la manière dont les acteurs vont construire dans leurs énonciations et mobiliser dans leurs pratiques une certaine vision du réel qui fait sens pour eux, qui justifie leurs prises de position et permet de déployer un discours critique. Pour ces acteurs médiatiques, au premier rang desquels se trouvent des journalistes politiques, cette opinion publique - régulièrement énoncée comme "un sentiment général ", " des grandes tendances " - existe bel et bien et doit faire l'objet d'un traitement journalistique.

Deux questionnements viennent animer notre réflexion.

1) Comment le triptyque équationnel entre opinion publique, internautes et réseaux socionumériques est-il construit dans la pratique journalistique?

2) Quelles sont les logiques et les intérêts qui sous-tendent et légitiment ce discours ?

Notre analyse vise à comprendre la manière dont les journalistes - bousculés dans leur identité, leur centralité et leurs pratiques professionnelles par les réseaux socionumériques (Mercier, PignardCheynel, 2014) - peuvent se saisir de propos recueillis en ligne en tant qu'éléments objectivables d'une opinion publique « connectée » en plus d'une opinion publique sondagière (Blondiaux, 1997) déjà instituée et légitimée.

Nous formulons l'hypothèse selon laquelle la mise en place de ces dispositifs intermédiatiques de récolte d'opinions vise à produire un nouveau régime de l'opinion publique (Gaïti, 2007) à moindre coût, fondé notamment sur un principe d'ubiquité et une mise en visibilité des saillances discursives et/ou relationnelles, qui offre au journaliste une plasticité énonciative lui permettant de croiser différents registres en passant de l'information à l'info-divertissement.

L'étude proposée prend appui sur l'émission politique télévisée Des Paroles et des actes entre le 24 septembre 2015 et le 26 mai 2016, soit six émissions contenant une séquence finale nouvelle dédiée aux réactions des internautes. Chaque séquence a fait l'objet d'une transcription intégrale manuelle 
avec captures d'écran, permettant de travailler à la fois les logiques discursives mais également les mises en scène visuelles. L'arrivée de ce type de dispositif dans une émission politique phare de la télévision française avec en moyenne 2.5 millions de téléspectateurs, est un signe de l'importance que prennent dans la pratique journalistique l'expression d'internautes sur les réseaux sociaux.

Nous allons voir que la séquence retenue cherche à dessiner, à croquer les grandes tendances des réactions de "Français " à l'émission, en mobilisant à la fois un registre de l'expertise, fondé sur une recherche de la vérité et de l'objectivité et un registre de l'info-divertissement, fondé sur une recherche de petites phrases dans une ambiance humoristique et critique.

\section{Croquer une opinion publique connectée : objectivation et expertise journalistique}

Jusqu'alors, l'émission diffusait une sélection de tweets, la plupart du temps sous forme de scroll en bas de l'écran, se superposant au débat, sans pour autant en prendre véritablement part. L'émission du 24 septembre 2015 propose pour la première fois, en plus de la diffusion continue de tweets qui prévalait jusqu'alors, une nouvelle séquence en fin d'émission, appelée "Le verdict », animée par Karim Rissouli, journaliste politique présenté comme « expert " des réseaux sociaux et Jean-Daniel Lévy, sondeur chez Harris Interactive.

L'intégration d'une séquence sondagière pour évaluer la personnalité politique invitée à l'émission n'est pas nouvelle. En 1985, l'émission L'Heure de vérité (1982-1995) ajoute une séquence à l'intérieur de laquelle est rendu public un sondage express par Minitel réalisé par la SOFRES auprès de 500 téléspectateurs. Ce « sondage-express » proposait une évaluation de la performance de l'invité politique pendant l'émission. Ce dispositif évaluatif, s'il n'est pas systématisé à l'ensemble des émissions politiques en direct, fait quand même partie, depuis plusieurs années, du répertoire des journalistes politiques en contexte télévisuel. Les professionnels des sondages, représentants d'instituts ont d'ailleurs acquis une place de choix dans les émissions politiques en étant régulièrement invités à la table du débat et dont la parole semble légitime et attendue. A l'instar du sondage, les énoncés produits à partir de tweets sont " provoqués, travaillés, encadrés, standardisés, simplifiés " (Blondiaux, 1997) et font ainsi l’objet d'une généralisation sans réelle remise en question alors même que les modalités de construction d'une " opinion publique " demeurent encore plus opaques que celles qui permettent la réalisation de sondage.

La séquence de Karim Rissouli cherche elle aussi à évaluer la prestation de l'invité politique, à partir d'une analyse des discours produits sur les réseaux socionumériques, notamment Twitter, plutôt que par le biais d'une analyse sondagière. Intégrer dans l'émission un journaliste dit « expert des réseaux sociaux " est nouveau. Dès lors, l'analyse des réseaux sociaux apparait comme aussi pertinente que la livraison des résultats d'un sondage. Ainsi, dans la pratique et le discours, viennent se juxtaposer deux types d'opinions publiques qui seraient complémentaires: l'une sondagière, et l'autre connectée.

Ce type de pratique journalistique qui vise à sélectionner sur les réseaux sociaux les énoncés discursifs jugés pertinents, les synthétiser et en rendre compte, commence à s'instituer dans certaines émissions qui traitent de politique ( $C$ politique sur France 5 avec la séquence de Bastien Hugues, $C Q F D$ sur Itélé avec Véronique Reille-Soult). L’arrivée d'une séquence dédiée aux tweets dans cette émission politique de renom marque sans doute une étape supplémentaire dans l’importance qu'accordent les journalistes à ce type de pratiques.

\section{Pluralité des objectifs et appréhension de Twitter comme espace protéiforme}

A chaque émission, David Pujadas et Karim Rissouli rappellent les objectifs de cette séquence. Nous pouvons en identifier quatre à partir des énonciations produites par les journalistes en plateau. Il 
s'agit tout d'abord de recenser et résumer les tweets publiés, c'est-à-dire de faire un travail de veille, de collecte et de traduction : "Karim, vous avez regardé, lu, analysé les messages, les tweets reçus ce soir" (Emission du 26.05.2016). Tout au long de l'émission, nous voyons d'ailleurs Karim Rissouli au premier rang du public, les yeux rivés sur un ordinateur. La mise en scène télévisuelle participe à produire une figure du journaliste connecté, expert des réseaux socionumériques. Le second objectif consiste pour le journaliste à dresser le portrait-robot de l'invité : "Est-ce qu’il y a une sorte de portrait-robot de François Bayrou qui est dressé ?" (Emission du 12.11.2015). Il s'agit ici de mettre en exergue une sélection de discours produits par des twittos qui pointent certains traits de caractère du personnage politique, tels que le courage, la probité, l'orgueil par exemple. Le portrait-robot ainsi dressé ne peut être envisagé comme l'expression subjective du journaliste mais comme un agrégat composite d'expressions singulières qui permettent une construction collective. Le journaliste prend d'ailleurs soin de se positionner comme médiateur voire traducteur d'une opinion publique connectée. Le troisième objectif vise à prononcer le verdict des internautes, transformant par là même l'espace scénique télévisuel en tribunal médiatique : Quel sentiment après votre prestation Jean-Luc Mélenchon ? Ceux qui nous regardent ont-ils été convaincus ?» (Emission du 26.05.2016). Le journaliste se charge non seulement de relayer certaines opinions et critiques formulées mais aussi de dire si la prestation de l'invité a été jugée positivement ou négativement par la majorité des twittos. Enfin, Karim Rissouli doit animer une séquence qui intègre aussi de l'autodérision, c'est-à-dire qui emprunte une caractéristique propre à l'info-divertissement: "Il ne faudra pas tout prendre au premier degré " (Emission du 24.09.2015). Une émission politique de grande écoute tente ici d'inclure pour la première fois un discours et des pratiques qui étaient jusqu'alors déconsidérées par les journalistes politiques.

En analysant les quatre objectifs prononcés à l'égard de cette séquence, nous notons une ambigüité intrinsèque quant à la position du journaliste, à la fois expert des réseaux socionumériques et amuseur public, mobilisant parfois même la caricature. Le journaliste doit composer une énonciation qui s’intéressera à la fois au général pour faire advenir une "opinion publique » et au particulier en rendant visibles des saillances, des "petites pépites » à partir de cette opinion publique : "Vous nous livrez les grandes tendances ou les petites pépites, ce qui a fait réagir, ce qui a fait rebondir." (Émission du 12.11.2015).

Enfin, les deux journalistes en plateau mobilisent une vision plurielle de Twitter, à la fois considéré comme le thermomètre d'une certaine opinion publique, un espace où la parole est à la fois libérée et passionnée (allant même jusqu'à parler de Twitter comme un "réceptacle d'insultes"), mais aussi un espace où la parole est contrôlée, vérifiée et maîtrisée (lieu de vérification des énoncés ou encore "énorme rédaction de journalistes" voire même tribunal d'opinion). Ce type d'appréhensions valorise en creux le besoin de faire appel à un journaliste pour effectuer une médiation, donner du sens à des dizaines de milliers d'énoncés.

\section{Le chiffre comme approbation d'un succès commercial et démocratique}

Chaque séquence commence systématiquement par un bilan du nombre de tweets produits pendant l'émission. Il s'agit de la première information délivrée par le journaliste à l'invité politique :

"Alors on commence avec une première info qui, elle, est incontestable. C'est le nombre de tweets que l'émission a généré ce soir. [...] On en était à 45000 tweets. 45000 tweets, c'est exactement la movenne pour ce type d'émission. Donc ça n'a pas sur-réagit, ça n’a pas sous-réagit. [...] Et on est loin du record d'Alain Juppé qui était autour de 58000 tweets. » (Émission du 24.09.2015)

"On est autour de 20000 tweets je pense, quoique ce que vous venez de dire va peut-être relancer la machine. Autour de 20000 tweets, c'est la moyenne basse de l'émission. " (Émission du 12.11.2015)

«Enormément de tweets! C'est plus que beaucoup! Peut-être que ça ne vous dit rien mais vous avez généré 86000 tweets au moment où on se parle. Record pour cette émission depuis qu'on a 
décidé de les comptabiliser. [...]86 000 ! Et au hasard pour comparer : Alain Juppé. Bon, c'était 57 000 tweets. [...] 86000 contre 57000 pour Juppé. » (Emission du 04.02.2016 avec Nicolas Sarkozy)

Karim Rissouli énonce des records, des comparaisons, des moyennes qui ne sont pas seulement mobilisés à titres indicatifs mais qui participent aussi à produire une énonciation sur la performance de l'émission et de l'invité. Plus le volume de tweets est élevé et plus la prestation de l'invité politique peut être considérée comme réussie par les journalistes. La stratégie de la comparaison permet d'ailleurs au journaliste de participer à la mise en scène de la compétition politique. Établir une comparaison des performances entre Nicolas Sarkozy et Alain Juppé n'est pas neutre dans la mesure où les deux invités politiques sont lancés dans la compétition des primaires pour l'élection présidentielle française de 2017. Dire à Nicolas Sarkozy qu'il a battu le record d'Alain Juppé permet d'entretenir un suspens en renforçant la compétition entre les deux favoris et la dimension spectaculaire de la politique.

On ne peut qu'être surpris par l'absence de rigueur dans l'appréhension des tweets. La moyenne de production de tweets par émission, énoncée par Karim Rissouli, est très largement fluctuante. Pendant la séquence avec Manuel Valls, Karim Rissouli énonce une moyenne de 45000 tweets par émission. Pendant la séquence avec François Bayrou, la moyenne passe à 20000 tweets (moyenne basse) et 32000 tweets pendant la séquence avec Mélenchon (moyenne basse également). Ainsi, énoncer une moyenne de production de tweets qui va de 20000 à 45000 tweets n'est ni crédible ni sérieux. Cela participe pourtant à nourrir le discours journalistique sur la participation du public au débat politique.

Cette évaluation n'est pas seulement celle du 'succès' de l'invité, mais aussi une manière de démontrer le succès de l'émission. Cet élément permet aussi de légitimer un succès commercial et démocratique, autant qu'il permet à l'émission de se valoriser elle-même, d'apparaitre comme légitime pour les invités.

\section{Le fact-checking ou la réaffirmation d'une autorité énonciative}

Chaque séquence comporte un temps consacré au fact-checking, soit à la vérification des affirmations de l'invité politique. La particularité de ce dispositif, qui se développe de plus en plus dans la pratique journalistique, réside dans le fait que le journaliste n'est pas forcément celui qui lance l'alerte, mais celui qui vérifie et relaye ou met en doute les informations produites par des hommes politiques ou des twittos.

Cette utilisation permet au journaliste de renégocier son statut vis-à-vis d'autres producteurs d'informations, en s'imposant comme le détenteur de la vérité. En effet, l'énonciation journalistique permet de repositionner le journaliste dans le rôle du vérificateur légitime, reléguant ainsi les affirmations de certains twittos comme potentiellement douteuses et nécessitant une vérification préalable :

«Vous le savez, les internautes, ils ne laissent absolument rien passer sur Twitter. L'une de vos informations a été particulièrement contestée ce soir. C'est quand vous parliez des fonctionnaires et vous avez dit: « les Français veulent plus de militaires pour les protéger. Et les militaires ce sont des fonctionnaires.". Plusieurs personnes nous ont dit: "Non, les militaires ne sont pas des fonctionnaires " notamment vetriana01. Alors qui a raison? On a vérifié. " (Émission du 24/09/15)

Le journaliste est ainsi mis en abîme, se proposant de "fact-checker" des fact-checkeurs, de proposer une vérité à partir de discours affirmant eux-mêmes une vérité. Procéder à un travail de fact-checking à partir des tweets produits par les internautes téléspectateurs permet à la fois au journaliste de donner une certaine forme de légitimité aux twittos, en relayant et en validant les affirmations qui peuvent être produites, mais aussi de se positionner comme intermédiaire voire même comme porte-parole d'une opinion publique connectée. 
Recourir au « micro-twittoir » : le journaliste comme porte-parole d'une vox populi

Si l'on analyse la manière dont les deux journalistes en plateau parlent des tweets référencés autour de l'émission, nous constatons un discours selon lequel chaque tweet publié sur le réseau est nécessairement adressé à l'émission, aux journalistes de l'émission :

«Plusieurs personnes nous ont dit [...] On nous a beaucoup beaucoup alerté sur Twitter [...] On a reçu des dizaines de milliers de messages. " (Émission du 24.09.2015)

«Que nous disent nos téléspectateurs sur les réseaux sociaux ? (Émission du 26.11.2015)

Il s'agit bien ici d'une distorsion de la réalité de production des tweets. Si les twittos réagissent à l'émission, c'est avant tout pour donner leur opinion sur le réseau, discuter et critiquer et non uniquement pour adresser un message aux journalistes de l'émission. Bien sûr, certains énoncés sont adressés mais quand une émission de 2 h30 reprend en moyenne 35 tweets sur un volume moyen de 35000 tweets, les chances d'intégrer le cadre télévisuel sont extrêmement faibles puisque $0.1 \%$ des tweets produits vont apparaitre dans le cadre de l'émission politique télévisée. En partant du principe que les tweets produits avec le hashtag \#DPDA auraient comme fonction première de s'adresser aux journalistes de l'émission, nous observons un effet centralisateur positionnant le journaliste non seulement comme relai mais aussi comme facteur du courrier numérique des téléspectateurs :

" Il y a du bon. Il y a du moins bon. Il y a du gentil. Il y a du beaucoup moins gentil. On a essayé de faire le tri. Et puis cet autre message un peu moins sympa qui résume le sentiment général : « Manuel Valls a-t-il vraiment besoin d'avoir tous ses ministres assis tranquillou derrière? Il emmène sa cour partout point d'interrogation? ". Ce n'est pas très sympa mais ça résume, je vous assure, le sentiment général sur cette opération de com ce soir sur Twitter. » (Émission du 24/09/15)

Dans cet extrait, le journaliste énonce l'existence d'un «sentiment général » dont il se fait le porteparole. A aucun moment, il ne va justifier le procédé à partir duquel il peut tirer cette conclusion. Sa position d'" expert " lui permet d'asseoir une autorité énonciative indépendamment de la réalité factuelle de l'énonciation. Pour renforcer cette impression de vérité, Karim Rissouli mobilise sa propre personne comme garante de la vérité. Avec la formulation "je vous assure ", il fait valoir sa grandeur et sa légitimité pour valider son propos. L’autorité énonciative dont il est porteur semble le préserver d'éventuelles contradictions que l'on pourrait lui faire. S'il reconnait ne pas pouvoir faire preuve de la même scientificité que son collègue sondeur, il se reconnait cependant la capacité d'évaluer les tweets et de proposer une quantification hors sol. A défaut de pouvoir produire des données chiffrées - en dehors du volume total de tweets produits - comme peut le faire un sondeur, le journaliste doit quand même proposer une énonciation quantitative qui va légitimer son propos: "Ça a été beaucoup commenté sur Twitter" (Émission du 21.01.2016), "Je vous assure que c'est beaucoup revenu.» (Émission du 04.02.2016). L'utilisation d'adverbes, tels que «beaucoup ", participe au discours évaluatif et critique du journaliste et le place en relai de cette « opinion publique connectée ». Ainsi, nous observons une stratégie d'effacement énonciatif du journaliste qui lui permet de livrer des critiques parfois dures tout en se dédouanant d'en être l'instigateur dans l'éventualité où l'invité politique s'en prendrait au journaliste. Cela se trouve illustré dans la séquence avec Jean-Luc Mélenchon qui remet en question cette stratégie du journaliste, et l'oblige ainsi à se justifier :

K. Rissouli : Est-ce que vous reconnaissez un peu cette petite mégalomanie ?

J.-L. Mélenchon : Non.

K. R. : Non?

J.-L. M. : Non.

K. R.: Pas du tout? Jamais?

J.-L. M.: Non. La mégalomanie, vous savez que c'est une maladie. Je ne me sens pas malade. (Rires du public) 
K. R.: Très bien.

J.-L. M.: Par contre, il y a une certaine mégalomanie à juger les autres et à leur attribuer des traits de caractère qu'ils n'ont pas.

K. R.: On essaie de regarder ce qui se dit de vous et je vous le restitue.

J.-L. M. : Oui, oui, c'est ça ! [...] Non mais quelques fois on ne sait pas! On a une opinion et on vous dit que vous êtes hargneux.

D. Pujadas : Ce n'est pas ce qu'on a dit!

J.-L. M. (désignant Karim Rissouli) : C'est ce qu'il a dit lui ! Vous ne suivez pas l'émission Pujadas ! (Rires du public)

D. P. : Un téléspectateur ! C'est ce qu'a dit un téléspectateur.

En définitive, cette séquence finale dans l'émission montre un double positionnement du journaliste qui pourrait apparaitre comme contradictoire au premier abord, mais qui semble parfaitement légitimé. D’une part, Karim Rissouli s'impose comme une figure d'autorité garante de la vérité notamment en utilisant l'outil du fact-checking pour réaffirmer des faits objectifs et vérifiables. D'autre part, cette autorité énonciative lui permet de proposer un discours sur Twitter et une analyse de tweets en faisant figurer une «opinion publique connectée » dont les modalités de construction sont encore plus opaques et moins scientifiques que celles des sondages : "Honnêtement je ne peux pas être aussi scientifique que Jean-Daniel ce soir." (Emission du 21.01.2016). Cette modalité de saisie des tweets revêt de nombreuses similitudes avec le dispositif du micro-trottoir déjà utilisé dans la pratique journalistique. Michelle Betz dans son Manuel des journalistes audiovisuels (2003) définit le micro-trottoir comme une "collection d'interviews très courtes avec un certain nombre de personnes, choisies habituellement de manière aléatoire (.../ pour introduire l'opinion des gens ordinaires dans une nouvelle audiovisuelle». C'est pour cette raison que nous pouvons parler de micro-twittoir au sens où le journaliste rend visible une sélection de réactions plurielles formulées dans un espace public et censées être représentatives d'un état d'esprit général sur une question donnée.

\section{Croquer le politique : de l'expertise à l'info-divertissement}

Nous venons de voir que le journaliste croque ce qu'il envisage comme étant une opinion publique en esquissant des grandes tendances, des traits généraux. Or, un autre enjeu émerge de ces pratiques. Il s'agit pour le journaliste de croquer l'invité(e) politique, c'est-à-dire de dépeindre son portrait mais aussi de le manger au sens symbolique du terme, de le dominer, de le déstabiliser, de jouer avec lui. La chronique mobilise alors des codes propres aux émissions de l'info-divertissement, cassant ainsi le caractère sérieux de l'émission.

\section{Le tweet comme petite phrase}

Nous avons observé que la médiation de tweets par le journaliste repose sur l'idée que ces derniers, sélectionnés pour être télédiffusés, incarnent, condensent, illustrent ce que pensent " les Français ". En ce sens, le tweet est érigé comme argument ou remarque d'autorité et s'intègre dans la stratégie d'assertion que mobilise le journaliste. En prétendant restituer une opinion générale, Karim Rissouli valide plus ou moins tacitement le déplacement de la singularité d'un tweet vers une opinion qui serait celle des internautes et plus largement celle des Français :

"Mais il y a un paradoxe quand même ce soir David, c'est que dans la posture il est jugé bon ministre de l'Intérieur. Mais que l'émission avançant ce soir, il n'a pas vraiment rassuré les Français. Ça, c'est beaucoup revenu aussi. » (Emission du 26.11.2015) 
Dans le cadre qui nous intéresse, le tweet peut être envisagé comme une "petite phrase " (Alice Krieg Planque, 2011) à la fois coproduit par le dispositif technique et l'énonciateur, remarquable, détachable, maitrisable et pouvant faire l'objet de reprises. Il s'intègre aux stratégies de communication politique de plus en plus orientées vers la recherche de la bonne formule et au cadre d'écriture journalistique qui vise une efficacité langagière. D'un point de vue pratique, la mobilisation de petites phrases permet au journaliste de fabriquer sa séquence de manière réactive et adaptée au format du programme. Dans la mesure où les tweets produits recouvrent une pluralité de registres énonciatifs, le journaliste peut s'en saisir à la fois comme argument d'autorité - le tweet comme révélateur d'une opinion publique - et comme élément de divertissement - le tweet comme énoncé singulièrement remarquable.

C'est à partir de ces tweets et de la manière dont ils sont transposés dans la chronique que la séquence va prendre une tonalité nouvelle, celle de l'autodérision et de l'humour qui inscrit pour la première fois l'émission dans une logique d'info-divertissement. Le tweet ne vise non plus seulement à nourrir le débat politique mais aussi à nourrir le spectacle télévisuel. En annonçant qu' « il ne faudra pas tout prendre au premier degré», le journaliste brouille l'usage qu'il fait des tweets.

Si la petite phrase est souvent perçue "sur le mode de la déploration" (Krieg Planque et Ollivier Yaniv, 2011), il en va autrement dans la séquence observée. La petite phrase a pour fonction de soutenir l'argumentaire critique du journaliste, de renforcer la logique de proximité avec le public par la complicité et la médiation, de répondre à l'impératif de brièveté et d'efficacité. Mais elle sert également, de par ses qualités humoristiques ou critiques, à alléger le caractère sérieux de l'émission en empruntant les codes de l'info-divertissement.

\section{Rejouer les codes de l'émission politique : mobilisation de l'humour}

Deux types d'humour peuvent être repérés. D'une part, le journaliste, à travers une attitude et un langage volontairement plus familiers, cherche à faire rire le public et les invités. La séquence intervient en fin d'émission, à la suite de deux heures de débat. La recherche d'une plus grande proximité avec le public en plateau et les téléspectateurs passe ici par un discours moins formel, moins conventionnel que le cadre dans lequel il s'exprime. D'autre part, le journaliste va sélectionner les tweets afin d'en faire apparaitre les plus remarquables à l'écran. Tous les tweets concernent l'invité politique et font l'objet d'une reprise par le journaliste sous forme de question adressée. Cela réclame de la personnalité politique qu'elle montre une bonne maîtrise des codes médiatiques et plus particulièrement qu'elle se soumette volontairement à une séquence publique d'autodérision voire même de dénigrement :

"Pour finir, on va se détendre un peu. (.../ On va vous mettre à contribution si vous êtes d'accord. On vous met à contribution? Aux Etats-Unis, Barack Obama a fait un carton, je ne sais pas si vous avez vu ça, en acceptant de lire lui-même à l'antenne face caméra des tweets le concernant, des tweets plus ou moins gentils voire carrément méchants. Manuel Valls, je vais donc avoir... Ils sont là, regardez. Dans ma main droite, le tweet très gentil. Il est là. Et dans ma main gauche, le tweet méchant, peut-être carrément méchant mais bon. Main droite, main gauche? Moi je vous conseille la gauche et le tweet méchant parce qu'on va encore dire que vous êtes de droite si vous prenez la main droite, donc... (Rires du public)» (Émission du 24/09/15)

Karim Rissouli fait ici référence à la séquence des "Mean tweets" d'Obama dans l'émission politique Jimmy Kimmel Live!, très populaire aux Etats-Unis. Avec Manuel Valls, il se trouve confronté à un interlocuteur qui ne souhaite pas participer à ce jeu et s'emporte face à ces pratiques journalistiques. Cette séquence revêt deux dimensions intéressantes. D'une part, elle oblige le journaliste à se justifier sur sa pratique en rappelant le cadre dans lequel il pense sa séquence et l'attitude attendue du responsable politique : «Manuel Valls, c'est une séquence d'autodérision donc faut se détendre, je vous l'avais dit." (Émission du 24/09/15). D’autre part, nous observons un 
rapport de force entre la personnalité politique qui exprime un refus et les journalistes en plateau qui tentent de garder la face et sous couvert de choix imposent à l'invité le déroulé de la séquence. En signifiant son refus, Manuel Valls crée une discontinuité dans la mise en scène télévisuelle. Or, la séquence doit avoir lieu. Avec ou sans le consentement de l'invité :

D. Pujadas : Le Premier ministre ne souhaite pas le lire. Mais vous pouvez le lire Karim!

K. Rissouli : Oui mais ça aurait été plus drôle dans sa voix.

D. P. : Ah oui mais...

(S'en suit la lecture du tweet critique puis un long développement de Manuel Valls sur les raisons qui le poussent à ne pas rentrer dans le jeu proposé. Vient enfin le moment de lire le tweet «gentil »)

D. P. : On va peut-être lire quand même le message bienveillant qui était adressé, Karim Rissouli.

[...]

K. R. : J'avais dit que je lirai les deux. Manuel Valls, droit dans ses bottes. Courageux, percutant, sans détour, réaliste, hashtag homme d'état". Vous voyez, je me suis fait engueuler et j’ai été sympa à la fin. (Rires du public)

Les journalistes manifestent ici un renversement du pouvoir énonciatif. Manuel Valls n'a pas d'autres alternatives que de se soumettre au choix des journalistes et de lire en direct les tweets critiques. S'il a pu tout au long de l'émission garder une certaine maîtrise du discours, cette séquence induit une autre configuration dans laquelle il doit nolens volens garder la face au sens goffmanien du terme c'est-à-dire en tant que valeur sociale positive (Goffman, 1974). Exercice d'autant plus difficile pour l'invité qui n’a pas connaissance au préalable du contenu de cette séquence fabriquée en temps réel.

La mobilisation du registre de l'humour et d'un langage plus familier, plus ordinaire au sein d'une émission politique marque la volonté d'ouvrir le format à des mises en scène plus hybrides, alternant le sérieux et le superficiel, sacralisant et désacralisant la parole et la personnalité politique invitée.

\section{Rituel d'inversion et mise à l'épreuve de l'invité politique}

Pierre Leroux et Philippe Riutort interrogent le rapport de pouvoir qu'il existe entre l'invité politique et le journaliste dans le rituel d'inversion : "Malgré un traitement souvent ironique et moqueur des animateurs, contribuant à retourner à leur profit le rapport de forces qui prévaut généralement entre journalistes et responsables politiques par l'établissement d'une sorte de "rituel d'inversion ", les invités politiques semblent accepter ce traitement qui leur est infligé en faisant preuve d'humour" (Leroux, Riutort, 2011). A la différence de l'émission politique qui ne remet pas toujours en question la sacralité du politique, les logiques de l'info-divertissement bousculent, voire même renversent la relation de pouvoir. Les journalistes peuvent ainsi mettre à l'épreuve le personnel politique en l'attaquant, en le critiquant, en le déstabilisant sur le terrain non seulement politique mais aussi personnel, et ce sous une apparente décontraction : "Le pire ennemi de Bayrou, c'est lui-même. Son ego est surdimensionné. " (Émission du 12.11.2015) «Mélenchon parle de lui à la troisième personne. Mégalo. » (Émission du 26.05.2016).

Cette séquence s'inscrit dans un mouvement beaucoup plus ancien de spectacularisation de l'émission politique et de désacralisation de l'invité politique (Lits, 2005 ; Leroux et Riutort, 2013). Elle vise à tester le politique non plus seulement sur ses compétences politiques mais aussi sur ses compétences médiatiques et communicationnelles. L'enjeu est d'apparaitre comme étant capable de faire preuve d'autocritique, de dérision, d'humour, de rondeur, de jeu d'esprits, de montrer son «autorité plastique» (Darras, 1997). Loin d'être anecdotique et superficielle, cette séquence constitue pour le politique un exercice à risque dans la mesure où elle participe à forger son image publique. Et c'est justement à partir de cet enjeu essentiel que le journaliste peut se permettre une prise de contrôle du discours et du face-à-face. En relayant des énoncés produits par une opinion 
publique « connectée », il fait advenir une critique renforcée par le poids du nombre et la force de l'assertion qu'il est alors difficile de critiquer.

\section{Conclusion}

En nous appuyant sur l'analyse de la séquence finale Des paroles et des actes, nous avons essayé de comprendre comment les journalistes politiques peuvent se saisir des tweets réactifs publiés lors de l'émission. Nous avons observé deux manières de mobiliser Twitter. D'une part, les tweets sont convoqués comme révélateurs d'une "opinion publique connectée " dont il s'agit de rendre compte. Mais ils sont d'autre part saisis dans leur dimension singulière, en tant que petites phrases. Ce traitement journalistique entraine un flou, une ambiguité, une hybridation de la pratique du journalisme politique qui alterne entre recherche d'objectivité, d'expertise, de généralités et mise en scène de singularités, de petites phrases, de l'anecdotique.

En intégrant des paroles nouvelles, dites citoyennes, dans l'agora télévisuelle, l'émission politique semble remplir sa fonction démocratique d'ouverture de la scène publique à des acteurs qui en étaient jusqu'alors exclus. Cependant, si l'ordre du discours (Foucault, 1971) peut sembler modifié, il n'en demeure pas moins que des logiques politiques de circulation et de contrôle de la production discursive semblent agir sur les formes de représentation de la parole citoyenne.

Ainsi, l'intermédiation telle qu'elle est mise en œuvre permet davantage de reproduire des rapports de pouvoir déjà institués avant même l'arrivée d'Internet et de mettre à profit les potentialités actancielles qu'il offre (Théviot, Mabi, 2014). La mise en scène de l'intermédiation procède d'une volonté d'intégration de réactions extérieures au cadre télévisuel tout en maintenant des formes visibles de différenciation entre les acteurs en présence, créant ainsi une tension entre engagement et distanciation, horizontalité et verticalité, mise en valeur et instrumentalisation.

L'intermédiation répond également aux exigences de proximité, de participation, de réactivité, de rythme et de plasticité qu'imposent aujourd'hui les cadres de l'émission politique (Leroux et Riutort, op. cit.). La séquence devient plus facile à fabriquer en ne réclamant plus une légitimité scientifique comme les sondages (Champagne, 1990). Si elle semble en apparence légère et accessoire, elle illustre plutôt bien l'émergence de pratiques journalistiques qui se fondent sur un triptyque équationnel pour fabriquer un nouveau registre d'opinion publique simple à fabriquer et à mobiliser.

Si l'intermédiation introduit dans le cadre de l'émission politique des éléments propres à l'infodivertissement qu'il semble aujourd'hui difficile de pouvoir contourner, elle peut être également pensée comme la marque d'une évolution durable des formats télévisuels vers une «intermédiation " stratégique dont cette séquence est une des manifestations possibles.

\section{Références bibliographiques}

Betz, Michelle (2003), Manuel des journalistes audiovisuels, Knight International Press Fellow.

Blondiaux, Loïc (1998), La fabrique de l'opinion : une histoire sociale des sondages, Paris : Seuil.

Blondiaux, Loïc (1997), Ce que les sondages font à l'opinion, Politix, vol. 10, n³7, p.117-136.

Boltanski, Luc, Thévenot, Laurent (1991), De la justification : les économies de la grandeur, Paris : Gallimard.

Boyadjian, Julien (2014), Twitter, un nouveau « baromètre de l'opinion publique "?, Participations, $\mathrm{n}^{\circ} 8, \mathrm{p} .55-74$. 
Champagne, Patrick (1990), Faire l'opinion. Le nouveau jeu politique, coll. Le sens commun, Paris : Editions de Minuit.

Darras, Éric (1997), Les bienséances de l'échange politique. Naissance d'une tribune politique télévisuelle, Politix, n³7, p.9-24.

Foucault, Michel (1971), L'ordre du discours, Paris : Gallimard.

Goffman, Erving (1974), Les rites d'interactions, Paris : les Editions de Minuit.

Krieg Planque, Alice, Ollivier Yaniv Caroline (2011), Poser les «petites phrases " comme objet d'étude, Communication \& Langages, n 168, p.17-22.

Krieg Planque, Alice (2011), Les 'petites phrases': un objet pour l'analyse des discours politiques et médiatiques, Communication \& Langages, n 168, p.23-41.

Leroux, Pierre, Riutort, Philippe (2013), La politique sur un plateau. Ce que la télévision fait à la représentation, Paris : PUF.

Leroux, Pierre, Riutort, Philippe (2011), Les " petites phrases " Les émissions de divertissement : de nouveaux lieux de valorisation des petites phrases ?, Communication \& langages, p.69-80.

Lits, Marc (2005), La spectacularisation des émissions politiques dans les télévisions européennes, Recherches en communication, n²4, p.7-18.

Mabi, Clément, Théviot, Anaiis (2014), Présentation du dossier. S’engager sur Internet. Mobilisations et pratiques politiques, Politiques de communication, n³, p.5-24.

Mercier, Arnaud, Pignard-Cheynel Nathalie (2014), Mutations du journalisme à l'ère du numérique : un état des travaux, Revue française des sciences de l'information et de la communication [en ligne], n̊5, 2014, mis en ligne le 17 juillet 2014, consulté le 12 juillet 2017, http://rfsic.revues.org/1097.

Neveu, Éric (1995), Les émissions politiques à la télévision. Les années quatre-vingt ou les impasses du spectacle politique, Hermès La Revue, n¹7-18, p.145-162. 